\title{
OPTIMAL RAMP METERING STRATEGY \\ WITH EXTENDED LWR MODEL, ANALYSIS AND COMPUTATIONAL METHODS
}

\author{
Denis Jacquet ${ }^{1}$ Carlos Canudas de Wit \\ Damien Koenig
}

\author{
Laboratoire d'Automatique de Grenoble \\ BP. 46, 38 402, St. Martin d'Hères, France
}

\begin{abstract}
This paper treats the problem of optimizing a coordinated ramp metering strategy based on a distributed macroscopic traffic model. The Lighthill, Whitham and Richards (LWR) model is extended to include on/off-ramps with on-ramp flow saturations and an optimal control formulation is proposed. Using optimization techniques in Banach spaces, a solution is shown to exist and the necessary optimality system is stated using formal adjoint calculus. An iterative descent algorithm and numerical methods for gradient evaluations are proposed to compute the optimal strategy with reasonable effort. The effectiveness of the approach is illustrated through a study case with field data. Copyright 2005 IFAC.
\end{abstract}

Keywords: optimal control, traffic control

\section{INTRODUCTION}

Because of the lack of space and limited public budgets, Intelligent Transportation Systems (ITS) have been considered as potential solutions to overcome the fast growth of peri-urban traffic volumes. Non-invasive ITS rely on traffic flow models, infrastructure monitoring devices (loop detectors, cameras, infrared sensors and radar) and infrastructure traffic management systems (ramp metering, variable speed limit, dynamic routing, user information) to control and ease traffic. Their purposes are to increase mobility and accessibility for residents and businesses by reducing congestion and crash rates. Ramp metering techniques are good candidates to improve the overall traffic flow in peri-urban area and benefit generally of user acceptance. Some control algorithms have been proposed using discrete (discretized macroscopic) traffic models (Papageorgiou et al., 1990; Hegyi et al., 2002) and several field tests

\footnotetext{
1 Corresponding author: denis.jacquet@lag.ensieg.inpg.fr
}

have been reported (Haj-Salem and Papageorgiou, 1995) with encouraging results. However, discretizations through Finite Difference Methods are known to alter essential phenomena in macroscopic traffic models (LeVeque, 1992) and may lead to inconsistent discrete versions. To overcome this problem, we propose in this paper to solve the ramp metering problem directly on the distributed model. Though discretizations are unavoidable to solve the optimality system, we hope the optimize-then-discretize approach to be more consistent and computationally efficient.

The traffic model proposed in this paper is an extension of the LWR model developed by Lighthill, Whitham and Richards (Whitham, 1974) that takes into account congestion propagation, boundary conditions, metered on-ramps, on-ramp flow saturation and off-ramp exit ratios. Ramps are modelled by an inhomogeneous term rather than boundary conditions for consistency. The coordinated ramp metering problem is casted, using an abstract and rigorous functional analysis set- 
ting, as an optimal control problem with a partial differential equation (PDE) constraint and control saturations. Assuming that the current traffic state, the boundary conditions and the exit ratios may be estimated/predicted and that queues are present at every on-ramps, the control objective is to compute, for a restricted ring road area and a finite time horizon, the optimal metering rates that maximize the aggregated flow (also known as vehicle-distance-travelled). Though this framework gives an open-loop strategy, a more robust closed-loop structure can be obtained by adding a feedback regulator layer or using Receding Horizon Control. Using optimization techniques in Banach spaces, necessary optimality conditions are stated and formal adjoint calculus is used to compute gradients with reasonable effort. Given the complexity of the optimality system, no closedform solution can be expected and an iterative gradient-based algorithm is designed to converge to a local optimum. This methodology postpones as further as possible the necessary discretizations (computers cannot handle infinite dimensional calculus). Then, the problem shifts from functional analysis to numerical analysis as the requirements become efficiency, robustness and accuracy of the numerical methods. A case study is fully treated and accurate discretization methods (LeVeque, 1992) are presented.

Optimization in functional spaces is a mature mathematical field which have been extensively treated the last three decades (Luenberger, 1969; Lions, 1971). Recently, this framework has proven to be well suited for the analysis and solving of optimal control problems involving distributed systems. Applications reported in the literature are fluid tracking (Hinze and Kunisch, 2001), vortex rebound control (Collis et al., 2001), robust fluid control (Bewley et al., 2000), aerospace design (Jameson et al., 1998) and optimal control of nonlinear conservation laws (Ulbrich, 2003). (Bayen et al., 2004) were the first to apply adjoint based gradient computation methods to traffic control in a boundary control framework.

Notations. $L_{\text {loc }}^{1}$ is the set of locally integrable functions and $C_{0}^{1}$ the set of continuously differentiable functions with compact support. For a function $\rho$ discontinuous on $\Gamma, \rho_{\Gamma} \overline{\text { and }} \rho_{\Gamma}^{+}$are its left and right values along $\Gamma$ and $[\rho]_{\mid \Gamma}=\rho_{\Gamma}^{+}-\rho_{\Gamma \Gamma}^{\bar{\Gamma}}$. For $X$ a Banach space with norm $\|\cdot\|_{X}, X^{*}$ is its topological dual and $\left\langle x^{*}, x\right\rangle_{X^{*}, X}$ is the duality pairing between $x^{*} \in X^{*}$ and $x \in X$. For a Hilbert space $X,\left\langle x_{1}, x_{2}\right\rangle_{X}$ is the inner product of $x_{1}$ and $x_{2}$ in $X \cdot \mathcal{L}(X, Y)$ is the set of linear operators from $X$ to $Y$. For $\mathcal{A}: X \rightarrow Y, D_{x} \mathcal{A}[\bar{x}] \in \mathcal{L}(X, Y)$ is its Fréchet derivative at $\bar{x}$ and $D_{x} \mathcal{A}[\bar{x}](\tilde{x})$ with $\tilde{x}$ small its Fréchet differential. $\mathcal{A}^{\star}$ is the formal adjoint of $\mathcal{A}$. Variables of integration are omitted in integrals and induced form context.

\section{EXTENDED LWR TRAFFIC MODEL}

The traffic infrastructure depicted in Figure 1 is considered for illustration purpose.

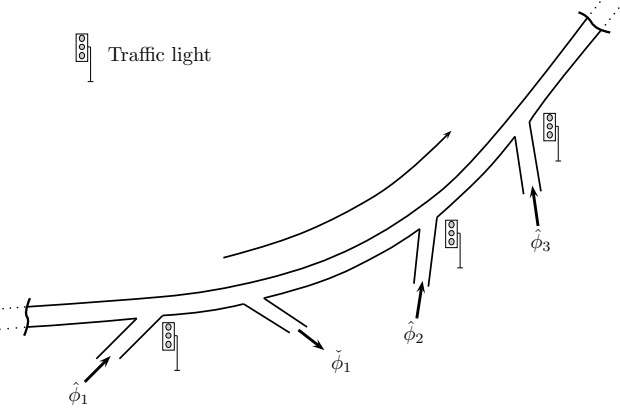

Fig. 1. Beltway section considered for illustration.

Macroscopic models are distributed systems where the traffic state is defined by aggregated quantities such as the vehicle density $\rho(t, x)$ in vehicles per unit length per lane and the mean speed $v(t, x)$. The simplest, involving $\rho$ only, is the LWR model proposed by Lighthill, Whitham (Whitham, 1974) and Richards. It is based on the vehicle conservation principle and the constitutive assumption that vehicles travel at the equilibrium speed $V(\rho)$, leading to the flow function $\Phi(\rho)=\rho V(\rho)$. Simple concave flow functions are the Greenshield (GS) and Greenberg (GB) models (Pipes, 1967)

$$
\Phi_{\mathrm{GS}}(\rho)=\rho \cdot v_{\mathrm{f}}-\frac{\rho^{2} \cdot v_{\mathrm{f}}}{\rho_{\mathrm{m}}} ; \Phi_{\mathrm{GB}}(\rho)=\rho \cdot v_{\mathrm{f}} \ln \left(\frac{\rho_{\mathrm{m}}}{\rho}\right)
$$

with free flow speed $v_{\mathrm{f}}$ and maximal density $\rho_{\mathrm{m}}$. The LWR model is extended to incorporate on/off-ramps with the following requirements:

R1 wave propagation phenomena similar to LWR, R2 on-ramp flows proportional to metering rates, R3 on-ramp flows saturated by main lane density, $\mathbf{R} 4$ density always less than maximal density $\rho_{\mathrm{m}}$.

A linear section is considered where $x \in[0, L]$, $\left\{\hat{x}_{1}, \ldots, \hat{x}_{N_{u}}\right\}$ are the locations of the $N_{u}$ metered on-ramps and $\left\{\check{x}_{1}, \ldots, \check{x}_{N_{w}}\right\}$ of the $N_{w}$ off-ramps. $u(t)=\left(u_{1}(t), \ldots, u_{N_{u}}(t)\right) \in[0,1]^{N_{u}}$ are the metering rates with on-ramp flows $\left(\hat{\phi}_{1}(t), \ldots, \hat{\phi}_{N_{u}}(t)\right)$ and $\left(w_{1}(t), \ldots, w_{N_{w}}(t)\right) \in[0,1]^{N_{w}}$ the exit ratios with off-ramp flows $\left(\check{\phi}_{1}(t), \ldots, \check{\phi}_{N_{u}}(t)\right)$.

To fulfil requirement $\mathbf{R} \mathbf{1}$, the modelling method used for the LWR formulation is adopted. For any restricted section as depicted in Figure 2,

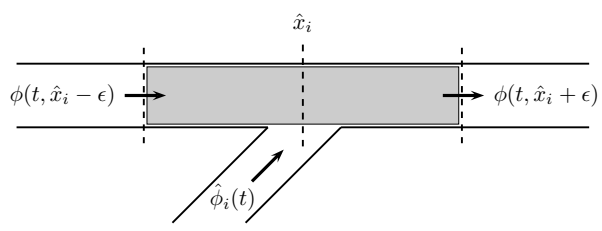

Fig. 2. Restricted section with one on-ramp. 
the vehicle conservation principle states that

$\partial_{t}[\mathrm{Nb}$ of vehicles $]=\sum[$ Inflows $]-\sum[$ Outflows $]$

$\partial_{t} \int_{\hat{x}_{i}-\epsilon}^{\hat{x}_{i}+\epsilon} \rho(t, \cdot)=\Phi\left(\rho\left(t, \hat{x}_{i}-\epsilon\right)\right)-\Phi\left(\rho\left(t, \hat{x}_{i}+\epsilon\right)\right)+\hat{\phi}_{i}(t)$

$\int_{\hat{x}_{i}-\epsilon}^{\hat{x}_{i}+\epsilon} \partial_{t} \rho(t, \cdot)=\int_{\hat{x}_{i}-\epsilon}^{\hat{x}_{i}+\epsilon}-\partial_{x} \Phi(\rho(t, \cdot))+\delta_{\hat{x}_{i}}(\cdot) \hat{\phi}_{i}(t)$

with $\delta_{\hat{x}_{i}}(x)$ the Dirac distribution centered at $\hat{x}_{i}$. Arbitrary $\hat{x}_{i}$ and $\epsilon$ lead to the divergence form

$$
\partial_{t} \rho+\partial_{x} \Phi(\rho)=\sum_{i=1}^{N_{u}} \delta_{\hat{x}_{i}} \hat{\phi}_{i}+\sum_{i=1}^{N_{w}} \delta_{\check{x}_{i}} \check{\phi}_{i}
$$

Requirements R2-R3-R4 are fulfilled by incorporating a each on-ramp a smooth saturation $\Psi_{i}(\cdot)$ as depicted in Figure 3. These saturations limit on-ramp flows for large main lane density by assuming $\hat{\phi}_{i}(t)=u_{i}(t) \Psi_{i}\left(\rho\left(t, \hat{x}_{i}\right)\right)$. Off-ramp flows are modelled by $\check{\phi}_{i}(t)=w_{i}(t) \Phi\left(\rho\left(t, \check{x}_{i}\right)\right)$.

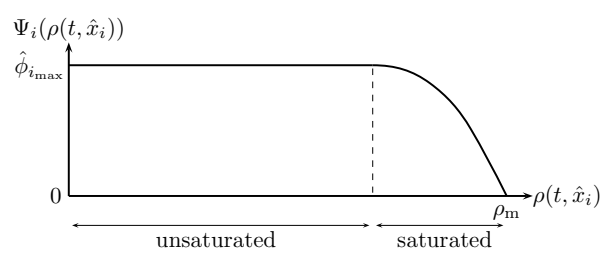

Fig. 3. Smooth saturation at on-ramp $i$.

To summarize, the proposed state equation is

$$
\partial_{t} \rho+\partial_{x} \Phi(\rho)=\sum_{i=1}^{N_{u}} \delta_{\hat{x}_{i}} u_{i} \Psi_{i}(\rho)-\sum_{i=1}^{N_{w}} \delta_{\check{x}_{i}} w_{i} \Phi(\rho)
$$

in $\Omega=(0, T) \times(0, L)$ with initial condition $\rho(0, x)=\rho^{I}(x)$ on $(0, L)$ and boundary conditions $\rho(t, 0)=\rho^{B_{u p}}(t)$ and $\rho(t, L)=\rho^{B_{d o}}(t)$ on $(0, T)$. For applications, functions $\Phi(\cdot)$ and $\Psi_{i}(\cdot)$ should be identified, initial condition estimated and exit ratios and boundary conditions predicted.

Equation (2) is a nonlinear hyperbolic conservation law, implying that $\rho$ may develop discontinuities. To be valid, it should be interpreted in the distributional or weak sense (Bressan, 2000), i.e.

$$
\begin{aligned}
& \int_{0}^{T} \int_{0}^{L} \rho \psi_{t}+\Phi(\rho) \psi_{x}+\int_{0}^{L} \rho^{I}(\cdot) \psi(0, \cdot)= \\
& \sum_{i=1}^{N_{w}} \int_{0}^{T} \psi\left(\cdot, \check{x}_{i}\right) \check{\phi}_{i}(\cdot)-\sum_{i=1}^{N_{u}} \int_{0}^{T} \psi\left(\cdot, \hat{x}_{i}\right) \hat{\phi}_{i}(\cdot)
\end{aligned}
$$

$\forall \psi \in C_{0}^{1}(]-\infty, T[\times] 0, L[)$, the appropriate space of test functions. Equation (2) must thus be interpreted in the dual space $\Lambda^{*}=C_{0}^{1^{*}}$ which has important implications for differentiation.

With the entropy inequality (Ansorge, 1990) as admissibility condition, the weak state equation (3) is well-posed (Bressan, 2000) and has a unique physical solution $\rho \in C\left((0, T), L_{\mathrm{loc}}^{1}((0, L))\right)$ that can be computed numerically (LeVeque, 1992). The admissibility condition states that a discontinuity $[\rho] \neq 0$ at $x=s(t)$ is allowed to propagate as a shock wave if $\rho^{-}<\rho^{+}$, the shock propagation speed being given by $\dot{s}(t)=[\Phi(\rho)] /[\rho]$.

Boundary conditions should be handled carefully to avoid an over-prescribed formulation. They apply smoothly upstream if $\Phi^{\prime}(\rho(t, 0))>0$ and downstream if $\Phi^{\prime}(\rho(t, L))<0$. When not applicable, shock waves may enter the domain if

$\frac{\Phi\left(\rho^{B u p}(t)\right)-\Phi(\rho(t, 0))}{\rho^{B} u p(t)-\rho(t, 0)}>0$ or $\frac{\Phi\left(\rho^{B} d o(t)\right)-\Phi(\rho(t, L))}{\rho^{B} d o(t)-\rho(t, L)}<0$.

To get a compact and rigorous system state equation, let introduce the Banach and Hilbert spaces $Y=\left\{\rho \in C\left((0, T), L_{\text {loc }}^{1}((0, L))\right) \mid \rho(0, x)=\rho^{I}(x)\right.$, $\left.\rho(t, 0)=\rho^{B_{u p}}(t), \rho(t, L)=\rho^{B_{d o}}(t)\right\}$

$U=L^{2}((0, T), \mathbb{R})^{N_{u}}$

$\Lambda^{*}=C_{0}^{1^{*}}$

and the nonlinear mapping $\mathcal{C}: Y \times U \rightarrow \Lambda^{*}$

$$
\begin{aligned}
\mathcal{C}(\rho, u)= & \partial_{t} \rho+\partial_{x} \Phi(\rho) \\
& -\sum_{i=1}^{N_{u}} \delta_{\hat{x}_{i}} u_{i} \Psi_{i}(\rho)+\sum_{i=1}^{N_{w}} \delta_{\check{x}_{i}} w_{i} \Phi(\rho)
\end{aligned}
$$

The state equation becomes $\mathcal{C}(\rho, u)=0$ in $\Lambda^{*}=C_{0}^{1^{*}}$.

\section{PROBLEM STATEMENT}

The optimal ramp metering problem we propose to solve is the maximization of the vehicledistance-travelled for a finite time horizon $(0, T)$ and a restricted road section $x \in(0, L)$. The cost functional $\mathcal{J}_{\text {obs }}: Y \rightarrow \mathbb{R}$ is thus the opposite of the aggregated flow on $(0, T) \times(0, L)$, leading to the infinite dimensional optimization problem

$$
\begin{aligned}
& \underset{\rho \in Y}{\operatorname{Min}} \mathcal{J}_{\text {obs }}(\rho)=-\int_{0}^{T} \int_{0}^{L} \Phi(\rho) \\
& \text { Subj. to }\left\{\begin{array}{l}
\mathcal{C}(\rho, u)=0 \\
u \in U_{\text {ad }}=L^{2}((0, T),[0,1])^{N_{u}}
\end{array}\right.
\end{aligned}
$$

$U_{\text {ad }}$ is a convex and compact subset of $U$ with constraints $0 \leq u_{i}(t) \leq 1$. As $U_{\text {ad }}$ is compact and $\mathcal{J}_{\text {obs }}(\rho)$ is bounded from below, continuous and twice Fréchet differentiable, (5) has a (maybe local) solution. To handle the constraints on $u$, the classical barrier method is used with

$$
\mathcal{J}_{\mathrm{bar}}^{M}(u)=-\frac{1}{M} \sum_{i=1}^{N_{u}} \int_{0}^{T} \ln \left(u_{i}\left(1-u_{i}\right)\right)
$$

leading to the augmented abstract problem

$$
\begin{aligned}
& \operatorname{Min}_{\substack{\rho \in Y \\
u \in U}} \mathcal{J}(\rho, u)=\mathcal{J}_{\text {obs }}(\rho)+\mathcal{J}_{\text {bar }}^{M}(u) \\
& \text { Subj. to } \quad \mathcal{C}(\rho, u)=0
\end{aligned}
$$

The solution of (6) will converge to that of (5) as $M \rightarrow \infty$ while keeping solutions of $(6)$ in $U_{\mathrm{ad}}$. 


\section{OPTIMALITY SYSTEM}

\subsection{Abstract problem}

As optimization theory in Banach spaces is independent of a particular application, we consider here the abstract problem

$$
\operatorname{Min}_{\substack{y \in \mathcal{Y} \\ u \in \mathcal{U}}} \mathcal{J}(y, u) \quad ; \quad \text { Subj. to } \quad \mathcal{C}(y, u)=0
$$

In this section, the existence of all mathematical objects is assumed and will be verified for the ramp metering problem. Assuming that there exists $\bar{y}$ and $\bar{u}$ such that $\mathcal{C}(\bar{y}, \bar{u})=0$, that $\mathcal{C}$ is continuously Fréchet differentiable ${ }^{2}$ in neighborhoods of $\bar{y}$ and $\bar{u}$ and that $D_{y} \mathcal{C}[\bar{y}, \bar{u}]$ is continuously invertible, the implicit function theorem states that $y=y(u)$ locally. Moreover, the sensitivity operator $D_{u} y[\bar{u}]$ is the unique solution of

$$
D_{y} \mathcal{C}[y(\bar{u}), \bar{u}] \circ D_{u} y[\bar{u}]+D_{u} \mathcal{C}[y(\bar{u}), \bar{u}]=0
$$

Under a uniqueness assumption, (7) can be replaced by the equivalent reduced problem

$$
\operatorname{Min}_{u \in U} \mathcal{J}_{\text {red }}(u) \triangleq \mathcal{J}(y(u), u)
$$

Assuming $\mathcal{J}_{\text {red }}(u)$ is Fréchet-differentiable, the necessary conditions for $\left(y^{*}, u^{*}\right)$ to be optimal are

$$
\left\{\begin{array}{l}
\mathcal{C}\left(y^{*}, u^{*}\right)=0 \\
D_{u} \mathcal{J}_{\text {red }}\left[u^{*}\right]=0
\end{array}\right.
$$

The Chain rule gives

$$
\begin{aligned}
\left\langle D_{u} \mathcal{J}_{\text {red }}[\bar{u}], \tilde{u}\right\rangle_{\mathcal{U}^{*}, \mathcal{U}}=\left\langle D_{u} \mathcal{J}[\bar{y}, \bar{u}], \tilde{u}\right\rangle_{\mathcal{U}^{*}, \mathcal{U}} \\
+\left\langle D_{y} \mathcal{J}[\bar{y}, \bar{u}], D_{u} y[\bar{u}](\tilde{u})\right\rangle_{\mathcal{Y}^{*}, \mathcal{Y}}
\end{aligned}
$$

with $D_{u} y[\bar{u}]$ the solution to the sensitivity equation (8). Using its formal adjoint ${ }^{3} D_{u} y[\bar{u}]^{\star}$

$$
\begin{aligned}
\left\langle D_{u} \mathcal{J}_{\text {red }}[\bar{u}], \tilde{u}\right\rangle_{\mathcal{U}^{*}, \mathcal{U}}=\left\langle D_{u} \mathcal{J}[\bar{y}, \bar{u}], \tilde{u}\right\rangle_{\mathcal{U}^{*}, \mathcal{U}} \\
+\left\langle D_{u} y[\bar{u}]^{\star} \circ D_{y} \mathcal{J}[\bar{y}, \bar{u}], \tilde{u}\right\rangle_{\mathcal{U}^{*}, \mathcal{U}}
\end{aligned}
$$

From (8), we have

$$
D_{u} y[\bar{u}]^{\star}=-D_{u} \mathcal{C}[\bar{y}, \bar{u}]^{\star} \circ\left(D_{y} \mathcal{C}[\bar{y}, \bar{u}]^{\star}\right)^{-1}
$$

leading to

$$
\begin{aligned}
& D_{u} \mathcal{J}_{\text {red }}[\bar{u}]=D_{u} \mathcal{J}[\bar{y}, \bar{u}] \\
& \quad-D_{u} \mathcal{C}[\bar{y}, \bar{u}]^{\star} \circ D_{y} \mathcal{C}[\bar{y}, \bar{u}]^{-\star} \circ D_{y} \mathcal{J}[\bar{y}, \bar{u}]
\end{aligned}
$$

To simplify the computation, the adjoint variable $\lambda=-D_{y} \mathcal{C}[\bar{y}, \bar{u}]^{-\star} \circ D_{y} \mathcal{J}[\bar{y}, \bar{u}]$ is introduced, splitting the derivative computation in two steps

$$
\begin{aligned}
& D_{y} \mathcal{C}[\bar{y}, \bar{u}]^{\star} \lambda=-D_{y} \mathcal{J}[\bar{y}, \bar{u}] \\
& D_{u} \mathcal{J}_{\text {red }}[\bar{u}]=D_{u} \mathcal{C}[\bar{y}, \bar{u}]^{\star} \lambda+D_{u} \mathcal{J}[\bar{y}, \bar{u}]
\end{aligned}
$$

\footnotetext{
2 The Fréchet derivative of $\mathcal{A}: X \rightarrow Y$ may be defined as the operator $D_{x} \mathcal{A}[\bar{x}] \in \mathcal{L}(X, Y)$ implied in the Taylor expansion $\mathcal{A}(\bar{x}+\tilde{x})=\mathcal{A}(\bar{x})+D_{x} \mathcal{A}[\bar{x}](\tilde{x})+o(\tilde{x})$ with $\|o(\tilde{x})\|_{Y}$ vanishing as $\|\tilde{x}\|_{X} \rightarrow 0$.

3 The formal adjoint of $\mathcal{A} \in \mathcal{L}(X, Y)$ is $\mathcal{A}^{\star} \in \mathcal{L}\left(Y^{*}, X^{*}\right)$ defined by $\langle\lambda, \mathcal{A}(x)\rangle_{Y^{*}, Y}=\left\langle\mathcal{A}^{\star}(\lambda), x\right\rangle_{X^{*}, X}$ with $\lambda \in Y^{*}$ the adjoint variable.
}

and giving an alternative to $(9)$. Necessary conditions for $\left(y^{*}, u^{*}\right)$ to be optimal is $\exists ! \lambda^{*}$ such that

$$
\left\{\begin{array}{l}
\mathcal{C}\left(y^{*}, u^{*}\right)=0 \\
D_{y} \mathcal{C}\left[y^{*}, u^{*}\right]^{*} \lambda^{*}=-D_{y} \mathcal{J}\left[y^{*}, u^{*}\right] \\
D_{u} \mathcal{C}\left[y^{*}, u^{*}\right]^{*} \lambda^{*}+D_{u} \mathcal{J}\left[y^{*}, u^{*}\right]=0
\end{array}\right.
$$

where SE stands for State Equation, AE for Adjoint Equation and DE for Decision Equation. Solving the optimality system (12) analytically is in general hopeless and an alternative is to develop an iterative gradient-based method that convergences to $u^{*}$. From the Riesz representation theorem, if $U$ is an Hilbert space, the gradient $\nabla_{u} \mathcal{J}_{\text {red }}[u]$ can be identified with the Fréchet derivative $D_{u} \mathcal{J}_{\text {red }}[u]$ given in (11). Note that, in general, the gradient expression depends on the definition of the inner product $\langle\cdot, \cdot\rangle_{U}$ which may be considered as a design parameter. Formal adjoint computation can thus be used to compute $\nabla_{u} \mathcal{J}_{\text {red }}[u]$ with reasonable effort. Moreover, it can be shown using the Lagrangian approach that the adjoint variable $\lambda$ can be interpreted as a Lagrange multiplier, leading to $\lambda \in \Lambda=C_{0}^{1}$.

\subsection{Application to the ramp metering problem}

To apply the results of the last section, partial Fréchet derivatives $D_{\rho} \mathcal{C}, D_{u} \mathcal{C}, D_{\rho} \mathcal{J}$ and $D_{u} \mathcal{J}$ are needed. A Taylor expansion of $\mathcal{C}$ and $\mathcal{J}$ around $(\bar{\rho}, \bar{u})$ with perturbations $\tilde{u} \in U$ and $\tilde{\rho}$ compatible with the definition of $Y$ gives

$$
\begin{aligned}
& D_{u} \mathcal{C}[\bar{\rho}, \bar{u}]=\left(-\delta_{\hat{x}_{1}} \Psi_{1}(\bar{\rho}), \ldots,-\delta_{\hat{x}_{N_{u}}} \Psi_{N_{u}}(\bar{\rho})\right) \\
& D_{\rho} \mathcal{C}[\bar{\rho}, \bar{u}](\tilde{\rho})=\partial_{t} \tilde{\rho}+\partial_{x} \Phi^{\prime}(\bar{\rho}) \tilde{\rho}-\sum_{i=1}^{N_{u}} \delta_{\hat{x}_{i}} \bar{u}_{i} \Psi_{i}^{\prime}(\bar{\rho}) \tilde{\rho} \\
& +\sum_{i=1}^{N_{w}} \delta_{\check{x}_{i}} w_{i} \Phi^{\prime}(\bar{\rho}) \tilde{\rho} \mid \begin{array}{l}
\tilde{\rho}(0, x)=0 \\
\tilde{\rho}(t, 0)=0 \\
\tilde{\rho}(t, L)=0
\end{array} \\
& D_{\rho} \mathcal{J}[\bar{\rho}, \bar{u}]=-\Phi^{\prime}(\bar{\rho}) \\
& D_{u} \mathcal{J}[\bar{\rho}, \bar{u}]=\frac{-1}{M}\left(\frac{1}{\bar{u}_{1}}-\frac{1}{1-\bar{u}_{1}}, \ldots, \frac{1}{\bar{u}_{N_{u}}}-\frac{1}{1-\bar{u}_{N_{u}}}\right)
\end{aligned}
$$

Constraints on $\tilde{\rho}$ are due to the definition of $Y$ and boundary conditions are applicable depending on the sign of $\Phi^{\prime}(\bar{\rho})$ at $x=0$ and $x=L$. The validity of $D_{\rho} \mathcal{C}[\bar{\rho}, \bar{u}]$ is proven by showing that

$$
\frac{\left\|\partial_{x} \frac{1}{2} \Phi^{\prime \prime}(\bar{\rho}) \tilde{\rho}^{2}\right\|_{C_{0}^{1 *}}}{\|\tilde{\rho}\|_{Y}} \underset{\tilde{\rho} \rightarrow 0}{\longrightarrow} 0
$$

with $\|\cdot\|_{C_{0}^{1 *}}$ the natural induced dual norm

$$
\|\xi\|_{C_{0}^{1 *}}=\sup \langle\xi, \psi\rangle_{C_{0}^{1 *}, C_{0}^{1}}, \forall \psi \in C_{0}^{1},\|\psi\|_{C_{0}^{1}} \leq 1
$$

The adjoint identity which is two integrations by parts here is used to compute $D_{\rho} \mathcal{C}[\bar{\rho}, \bar{u}]^{\star}$ with the associated constraints on the dual variable $\lambda$. It can be shown that 


$$
\begin{gathered}
D_{\rho} \mathcal{D}[\bar{\rho}, \bar{u}]^{\star}(\lambda)=-\partial_{t} \lambda-\Phi^{\prime}(\bar{\rho}) \partial_{x} \lambda \\
-\sum_{i=1}^{N_{u}} \delta_{\hat{x}_{i}} \bar{u}_{i} \Psi_{i}^{\prime}(\bar{\rho}) \lambda+\sum_{i=1}^{N_{w}} \delta_{\check{x}_{i}} w_{i} \Phi^{\prime}(\bar{\rho}) \lambda \mid\left\{\begin{array}{l}
\lambda(T, x)=0 \\
\lambda(t, 0)=0 \\
\lambda(t, L)=0 \\
\lambda_{\Gamma}=0
\end{array}\right.
\end{gathered}
$$

Constraints on $\lambda$ set boundary terms from the integrations by parts to 0 with $\Gamma$ the set of points where $\bar{\rho}$ is discontinuous.

$D_{u} \mathcal{C}[\bar{\rho}, \bar{u}] \in \mathcal{L}\left(U, \Lambda^{*}\right)$ being an extension operator with Dirac measures, $D_{u} \mathcal{C}[\bar{\rho}, \bar{u}]^{\star}$ is the restriction

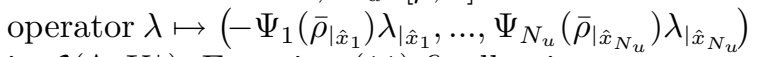
in $\mathcal{L}\left(\Lambda, U^{*}\right)$. Equation (11) finally gives

$$
\begin{aligned}
& D_{u} \mathcal{J}[\rho(\bar{u}), \bar{u}]= \\
& \left(\begin{array}{c}
-\Psi_{1}\left(\bar{\rho}\left(\cdot, \hat{x}_{1}\right)\right) \lambda\left(\cdot, \hat{x}_{1}\right)-\frac{1}{M}\left(\frac{1}{\bar{u}_{1}}-\frac{1}{1-\bar{u}_{1}}\right) \\
\vdots \\
-\Psi_{N_{u}}\left(\bar{\rho}\left(\cdot, \hat{x}_{N_{u}}\right)\right) \lambda\left(\cdot, \hat{x}_{N_{u}}\right)-\frac{1}{M}\left(\frac{1}{\bar{u}_{N_{u}}}-\frac{1}{1-\bar{u}_{N_{u}}}\right)
\end{array}\right)
\end{aligned}
$$

The optimality system for the ramp metering problem can be stated as $\left(\rho^{*}, u^{*}\right) \in Y \times U$ optimal for (6) if there exist a unique $\lambda^{*} \in \Lambda$ such that

$$
\left\{\begin{array}{l}
\partial_{t} \rho^{*}+\partial_{x} \Phi\left(\rho^{*}\right)=\sum_{i=1}^{N_{u}} \delta_{\hat{x}_{i}} u_{i}^{*} \Psi_{i}\left(\rho^{*}\right)-\sum_{i=1}^{N_{w}} \delta_{\check{x}_{i}} w_{i} \Phi\left(\rho^{*}\right) \\
\rho(0, x)=\rho^{I}(x) \\
\rho(t, 0)=\rho^{B u p}(t), \rho(t, L)=\rho^{B_{d o}}(t) \text { when applicable } \\
-\partial_{t} \lambda^{*}-\Phi^{\prime}\left(\rho^{*}\right) \partial_{x} \lambda^{*}=\Phi^{\prime}\left(\rho^{*}\right)+\sum_{i=1}^{N_{u}} \delta_{\hat{x}_{i}} u_{i}^{*} \Psi_{i}^{\prime}\left(\rho^{*}\right) \lambda^{*} \\
-\sum_{i=1}^{N_{w}} \delta_{\check{x}_{i}} w_{i} \Phi^{\prime}\left(\rho^{*}\right) \lambda^{*} \\
\lambda(T, x)=0, \lambda_{\Gamma}=0 \quad \lambda(t, L)=0 \text { if } \Phi^{\prime}\left(\left.\rho^{*}\right|_{L}\right)>0 \\
\lambda(t, 0)=0 \text { if } \Phi^{\prime}\left(\rho^{*}{ }_{0}\right)<0, \lambda\left(\frac{1}{M}\left(\frac{1}{u_{i}^{*}}-\frac{1}{1-u_{i}^{*}}\right)=0, \forall i\right.
\end{array}\right.
$$

The first three lines are the state equations, the next three the adjoint equations and the last ones the decision equations. Solving (13) analytically appear to be out of reach and we turn to the design of an iterative descent method.

As $U$ is an Hilbert space, $U^{*}=U$ and choosing the standard inner product in $L^{2}((0, T), \mathbb{R})^{N_{u}}$, the gradient $\nabla_{u} \mathcal{J}[\rho(\bar{u}), \bar{u}]$ may be identified with the Fréchet derivative $D_{u} \mathcal{J}[\rho(\bar{u}), \bar{u}]$ by the Riesz representation theorem. The gradient of $\mathcal{J}(\rho(u), u)$ may then be evaluated in three steps:

(i) With $\rho^{I}(x), \rho^{B_{\mathrm{up}}}(t), \rho^{B_{\mathrm{do}}}(t)$ and $w(t)$, compute $\rho(u) \in Y$ from the state equation.

(ii) Compute the adjoint variable $\lambda \in \Lambda$ from the adjoint equation which is a backward, inhomogeneous, linear, space and time varying advection Cauchy problem that depends on $u$ and $\rho(u)$. It is well posed even for discontinuous $\rho$ thanks to the admissibility condition as characteristics do not intersect backwards.

(iii) Compute $\nabla_{u} \mathcal{J}[\rho(u), u]$ with $\mathrm{i}^{\text {th }}$ component $\nabla_{u_{i}} \mathcal{J}[\rho(u), u]=-\Psi_{i}\left(\rho\left(\cdot, \hat{x}_{i}\right)\right) \lambda\left(\cdot, \hat{x}_{i}\right)-\frac{1}{M}\left(\frac{1}{u_{i}}-\frac{1}{1-u_{i}}\right)$

\section{COMPUTATIONAL ALGORITHM AND NUMERICAL EXPERIMENT}

If infinite dimensional computations were possible, the following algorithm solves (5) iteratively.

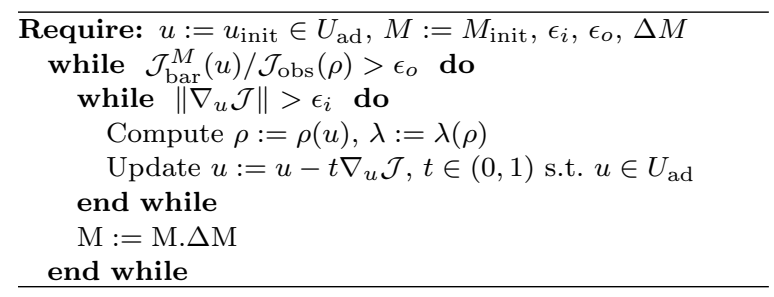

As all computations in $Y, U$ and $\Lambda$ cannot be done in a computer, numerical approximations are unavoidable. The state equation is a scalar inhomogeneous nonlinear hyperbolic conservation law, which makes its numerical resolution difficult. We propose to use the Godunov scheme (LeVeque, 1992) for its elegant interpretation, its good shock capturing capabilities (no artificial diffusion nor oscillation) and its treatment of boundary conditions. The computational domain approximating $Y$ is the uniform grid $\left\{\rho_{i}^{n}\right\}_{i=1, \ldots, N}^{n=1, \ldots, M}$ with periods $\Delta t$ and $\Delta x$. With the approximation $\rho_{i}^{0}=\int_{x_{i}-1 / 2}^{x_{i}+1 / 2} \rho^{I}(x)$, Godunov time stepping is

$\rho_{i}^{n+\frac{1}{2}}=\rho_{i}^{n}+\frac{\Delta t}{\Delta x}\left(\Phi\left(\rho_{i-\frac{1}{2}}^{*}\right)-\Phi\left(\rho_{i+\frac{1}{2}}^{*}\right)\right)$

$\rho_{i}^{n+1}=\rho_{i}^{n+\frac{1}{2}}+\frac{\Delta t}{\Delta x}\left(u_{\hat{\delta}(i)}^{n} \Psi_{\hat{\delta}(i)}\left(\rho_{i}^{n+\frac{1}{2}}\right)-w_{\check{\delta}(i)}^{n} \Phi\left(\rho_{i}^{n+\frac{1}{2}}\right)\right)$

where $\hat{\delta}$ and $\check{\delta}$ map spacial indices to on/off-ramp indices when applicable. $\rho_{i+\frac{1}{2}}^{*}$ is given by

\begin{tabular}{ll}
\hline If... & $\ldots$ then \\
\hline$\Phi^{\prime}\left(\rho_{i}^{n}\right) \geq 0, \Phi^{\prime}\left(\rho_{i+1}^{n}\right) \geq 0$ & $\rho_{i+\frac{1}{2}}^{*}=\rho_{i}^{n}$ \\
$\Phi^{\prime}\left(\rho_{i}^{n}\right)<0, \Phi^{\prime}\left(\rho_{i+1}^{n}\right) \leq 0$ & $\rho_{i+\frac{1}{2}}^{*}=\rho_{i+1}^{n}$ \\
$\Phi^{\prime}\left(\rho_{i}^{n}\right) \geq 0, \Phi^{\prime}\left(\rho_{i+1}^{n}\right)<0$ & $s=\frac{\Phi\left(\rho_{i+1}^{n}\right)-\Phi\left(\rho_{i}^{n}\right)}{\rho_{i+1}^{n}-\rho_{i}^{n}}$ \\
& $\rho_{i+\frac{1}{2}}^{*}=\rho_{i}^{n}, s \geq 0$ \\
& $\rho_{i+\frac{1}{2}}^{*}=\rho_{i+1}^{n}, s<0$ \\
$\Phi^{\prime}\left(\rho_{i}^{n}\right)<0, \Phi^{\prime}\left(\rho_{i+1}^{n}\right)>0$ & $\Phi^{\prime}\left(\rho_{i+\frac{1}{2}}^{*}\right)=0$ \\
\hline
\end{tabular}

The following hybrid Upwind/Downwind scheme is proposed for the adjoint equation

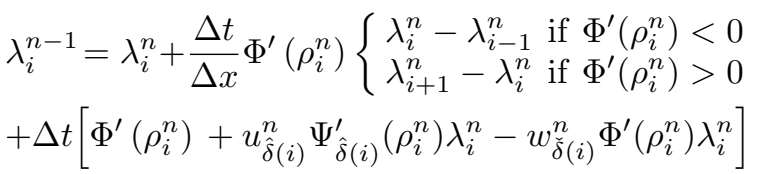

Both schemes require $\Delta x / \Delta t>\max \left|\Phi^{\prime}(\rho)\right|$ to have a stable convective part. Runge-Kutta methods may be necessary to stabilize the inhomogeneous terms. With $\iota(i)$ the index corresponding to the $\mathrm{i}^{\text {th }}$ on-ramp, the gradient is evaluated as

$$
\left(\nabla_{u_{i}} \mathcal{J}\right)^{n}=-\Psi_{i}\left(\rho_{\iota(i)}^{n}\right) \lambda_{\iota(i)}^{n}-\frac{1}{M}\left(\frac{1}{u_{\iota(i)}^{n}}-\frac{1}{1-u_{\iota(i)}^{n}}\right)
$$

As a numerical experiment, the maximization of the vehicle-distance-travelled for the network of 12 $\mathrm{km}$ in Figure 1 is treated. A time horizon of 1.5 hours at the beginning of the afternoon rush hours is considered with real field initial and boundary 
data courtesy of DDE Isère. Table 1 gives the simulation parameters and results, Figure 4 shows the iterations of the observation and augmented costs and Figure 5 the optimal on-ramp flows and the distributed flow improvement in $(0, T) \times(0, L)$.

\begin{tabular}{ll}
\hline simulation parameters & \\
\hline Number of space points & 150 for $12 \mathrm{~km}$ \\
Number of time points & 2700 for $1.5 \mathrm{~h}$ \\
Total number of points & 675000 \\
$v_{\mathrm{f}}$ form least square fitting of $\Phi_{\mathrm{GS}}$ & $109 \mathrm{~km} / \mathrm{h}$ \\
$\rho_{\mathrm{m}}$ form least square fitting of $\Phi_{\mathrm{GS}}$ & $75 \mathrm{veh} / \mathrm{km}$ \\
Optimization computational time & $35 \mathrm{~s}$ \\
Number of outer iterates & 12 \\
Last relative $\mathcal{J}_{\text {obs variation }}$ & $5.6695 \mathrm{e}-007$ \\
Last relative $\mathcal{J}$ variation & $-2.3012 \mathrm{e}-005$ \\
Last $\mathcal{J}_{\text {bar }}$ & -0.4661 \\
Last $\mathcal{J}_{\text {obs }}$ & $-1.1870 \mathrm{e}+005$ \\
\hline
\end{tabular}

Table 1. Simulation parameters.

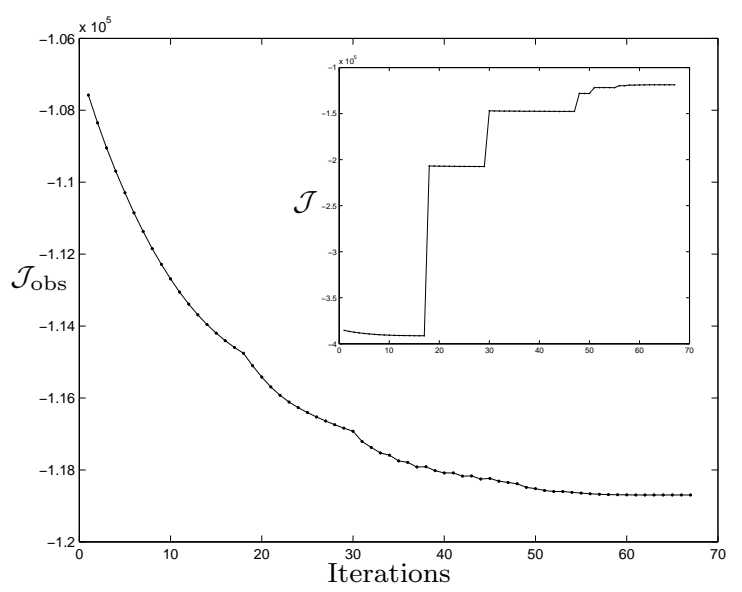

Fig. 4. Reductions of costs $\mathcal{J}_{\text {obs }}$ and $\mathcal{J}$.

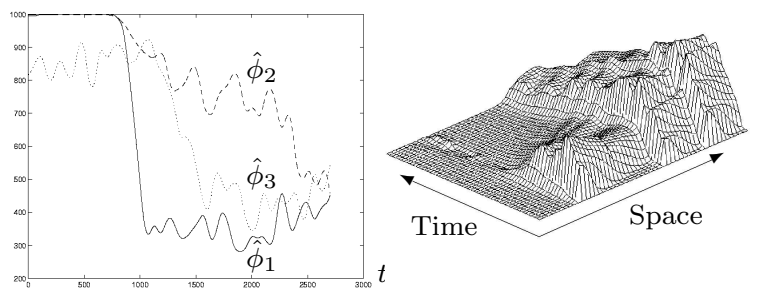

Fig. 5. Optimal on-ramp flows and distribution of the flow improvement in time and space.

\section{CONCLUSION}

This paper solves the problem of optimizing a coordinated ramp metering strategy with a distributed dynamical traffic model. We believe this optimize-then-discretize method to be more consistent than discretize-then-optimize approaches as macroscopic models are inherently distributed. Based on an infinite dimensional optimality system, numerical methods are proposed to compute iteratively and at reasonable cost the optimal strategy. A numerical experiment illustrates the method effectiveness. The introduced formalism allows with slight modification other choices for the cost functional and the traffic model.

\section{REFERENCES}

Ansorge, R. (1990). What does the entropy condition mean in traffic flow theory?. Transportation Research Part B.

Bayen, A. M., R. Raffard and C. J. Tomlin (2004). Network congestion alleviation using adjoint hybrid control: Application to highways. In: HSCC, rth Int. Workshop. Springer.

Bewley, T.R., R. Temam and M. Ziane (2000). A general framework for robust control in fluid mechanics. Physica D.

Bressan, A. (2000). Hyperbolic Systems of Conservation Laws - The One-dimensional Cauchy Problem. Oxford University Press.

Collis, S. S., K. Ghayour, M. Heinkenschloss, M. Ulbrich and S. Ulbrich (2001). Numerical solution of optimal control problems governed by the compressible Navier-Stokes equations. In: Optimal Control of Complex Structures, Int. Conf. in Oberwolfach. Birkhäuser.

Haj-Salem, H. and M. Papageorgiou (1995). Ramp metering impact on urban corridor traffic: Field results. Transportation Research Part A.

Hegyi, A., B. De Schutter, H. Hellendoorn and T. van den Boom (2002). Optimal coordination of ramp metering and variable speed control - an MPC approach. In: Proceedings of the 2002 American Control Conference.

Hinze, M. and K. Kunisch (2001). Second order methods for optimal control of timedependent fluid flow. SIAM Journal on Control and Optimization.

Jameson, A., L. Martinelli and N.A. Pierce (1998). Optimum aerodynamic design using the navier-stokes equations. Theoretical Computational Fluid Dynamics.

LeVeque, R. J. (1992). Numerical Methods for Conservation Laws. Birkhäuser.

Lions, J.L. (1971). Optimal control of systems governed by partial differential equations. Springer.

Luenberger, D. G. (1969). Optimization by Vector Space Methods. John Wiley \& Sons.

Papageorgiou, M., J.M. Blosseville and H. HajSalem (1990). Modelling and real-time control of traffic flow on the southern part of boulevard périphérique in Paris. part II: Coordinated on-ramp metering. Transportation Research Part A.

Pipes, L.A. (1967). Car following models and the fundamental diagram of road traffic. Transportation Research.

Ulbrich, S. (2003). Adjoint-based derivative computations for the optimal control of discontinuous solutions of hyperbolic conservation laws. Systems \& Control Letters.

Whitham, G.B. (1974). Linear and Nonlinear Waves. Pure and applied mathematics. John Wiley \& Sons. 